\title{
Diffusion-Weighted MRI for Selection of Complete Responders After Chemoradiation for Locally Advanced Rectal Cancer: A Multicenter Study
}

\author{
Doenja M. J. Lambregts, $\mathrm{MD}^{1,2}$, Vincent Vandecaveye, $\mathrm{MD}^{3}$, Brunella Barbaro, $\mathrm{MD}^{4}$, Frans C. H. Bakers, $\mathrm{MD}^{1}$, \\ Maarten Lambrecht, $\mathrm{MD}^{5}$, Monique Maas, $\mathrm{MD}^{1,2}$, Karin Haustermans, $\mathrm{MD}^{5}$, Vincenzo Valentini, $\mathrm{MD}^{6}$, \\ Geerard L. Beets, $\mathrm{MD}^{2}$, and Regina G. H. Beets-Tan, $\mathrm{MD}^{1}$ \\ ${ }^{1}$ Department of Radiology, Maastricht University Medical Center, Maastricht, The Netherlands; ${ }^{2}$ Department of Surgery, \\ Maastricht University Medical Center, Maastricht, The Netherlands; ${ }^{3}$ Department of Radiology, University Hospitals \\ Leuven, Leuven, Belgium; ${ }^{4}$ Department of Radiology, Università Cattolica del Sacro Cuore, Rome, Italy; ${ }^{5}$ Department of \\ Radiation Oncology, University Hospitals Leuven, Leuven, Belgium; ${ }^{6}$ Department of Radiation Oncology, Università \\ Cattolica del Sacro Cuore, Rome, Italy
}

\begin{abstract}
Purpose. In 10-24\% of patients with rectal cancer who are treated with neoadjuvant chemoradiation, no residual tumor is found after surgery (ypT0). When accurately selected, these complete responders might be considered for less invasive treatments instead of standard surgery. So far, no imaging method has proven reliable. This study was designed to assess the accuracy of diffusion-weighted MRI (DWI) in addition to standard rectal MRI for selection of complete responders after chemoradiation.

Methods. A total of 120 patients with locally advanced rectal cancer from three university hospitals underwent chemoradiation followed by a restaging MRI (1.5T), consisting of standard T2W-MRI and DWI (b0-1000). Three independent readers first scored the standard MRI only for the likelihood of a complete response using a 5-point confidence score, after which the DWI images were added and the scoring was repeated. Histology (ypT0 vs. ypT1-4) was the standard reference. Diagnostic performance for selection of complete responders and interobserver agreement were compared for the two readings.

Results. Twenty-five of 120 patients had a complete response (ypT0). Areas under the ROC-curve for the three readers improved from $0.76,0.68$, and 0.58 , using only
\end{abstract}

(C) The Author(s) 2011. This article is published with open access at Springerlink.com

First Received: 17 November 2010;

Published Online: 23 February 2011

R. G. H. Beets-Tan, MD

e-mail: r.beets.tan@mumc.nl standard MRI, to $0.8,0.8$, and 0.78 after addition of DWI $(P=0.39,0.02$, and 0.002$)$. Sensitivity for selection of complete responders ranged from $0-40 \%$ on standard MRI versus $52-64 \%$ after addition of DWI. Specificity was equally high (89-98\%) for both reading sessions. Interobserver agreement improved from $\kappa 0.2-0.32$ on standard MRI to $0.51-0.55$ after addition of DWI.

Conclusions. Addition of DWI to standard rectal MRI improves the selection of complete responders after chemoradiation.

The introduction of preoperative, rather than postoperative, adjuvant chemoradiation therapy (CRT) has led to a reduction in local recurrence rates and has become standard of care for patients with locally advanced rectal cancer. ${ }^{1}$ In $10-24 \%$ of patients, no residual tumor is found at histology after surgery. ${ }^{2}$ These complete responders are known to have a very good prognosis, in terms of overall and disease-free survival. ${ }^{2}$ A complete response also raises the hotly debated question of whether surgery is still necessary for these patients, especially because total mesorectal excision (TME) may have associated morbidity and even mortality and has the potential risk of a permanent colostomy. Recently, a more conservative treatment is advocated in patients who show a good or complete response to neoadjuvant treatment. In 2006, Habr-Gama et al. presented the long-term results of a prospective trial that investigated a "wait-and-see" policy in a carefully selected group of patients with clinical and radiological evidence of a complete response after neoadjuvant CRT. 
Results at 5-year follow-up were favorable for the nonsurgical group, with an overall and disease-free survival of $93 \%$ and $85 \%$, respectively. ${ }^{3}$ To safely omit surgery, it is essential to select accurately the right candidates, i.e., the true complete responders. This selection is mainly performed using digital examination, endoscopy, and biopsy, but these methods are not infallible. The role of imaging for restaging after CRT has been the subject of several studies and all suggest that neither MRI nor endorectal ultrasound or ${ }^{18} \mathrm{~F}$-fluorodeoxyglucose-positron emission tomography (FDG-PET) are sufficiently accurate for identifying the true complete responders with positive predictive values ranging from $17-50 \% .^{4-9}$ The use of these modalities for selection of patients would consequently put them at risk for undertreatment.

Diffusion-weighted MRI (DWI) is a functional MR imaging technique that uses differences in the extracellular movement of water protons to discriminate between tissues of varying cellularity. In tissues with normal cellularity, water protons can diffuse relatively freely, which results in a loss of signal on DWI. Conversely, in tissues with increased cellularity (tumor), the diffusion of water is restricted, resulting in remaining high signal on DWI. In many reports, DWI has shown promise for identification of malignant tumors, and recent studies on rectal cancer have indicated that DWI also may be useful for response evaluation after chemoradiation treatment. ${ }^{8,10-15}$ In 2009, Kim et al. showed in a study of 40 patients that DWI in addition to standard MRI significantly improved the performance of radiologists to select complete responders compared with standard MRI only. ${ }^{8}$

The purpose of our study was to evaluate the accuracy of DWI in addition to a standard restaging MRI for selection of complete responders after chemoradiation for locally advanced rectal cancer in a larger and multicenter study setting.

\section{METHODS AND MATERIALS}

\section{Patients}

This study retrospectively evaluated 120 consecutive patients who were treated for locally advanced rectal cancer in three university hospitals between 2005 and 2009. Due to the retrospective nature of the study, informed consent was not required. Ninety-three patients were men and 27 were women. Median age was 67 (range 22-89) years. Inclusion criteria consisted of (1) biopsy-proven rectal cancer, (2) locally advanced disease as determined on primary staging MRI (T3-4 tumor, tumor involvement of the mesorectal fascia, and/or positive nodal status), (3) preoperative treatment consisting of a long course of neoadjuvant chemoradiation treatment, and (4) availability of posttreatment MR imaging, including DWI. Exclusion criteria consisted of (1) nonresectable disease and (2) insufficient MR image quality (e.g., due to metal or motion artefacts). All patients underwent a long course of preoperative chemoradiation, consisting of capecitabine and/or oxaliplatin, combined with 50.4-55 Gy of radiation. After a 5-10-week time interval, all patients underwent a second, restaging MRI, including DWI, for response evaluation. Patients were then referred for further treatment.

\section{MR Imaging}

In each participating center, imaging was performed at 1.5T using a phased array body coil. The MR protocol consisted of standard T2-weighted fast spin echo sequences (as described in literature) in three orthogonal directions: sagittal, axial, and coronal with an in plane resolution ranging from $0.42-2.56 \mathrm{~mm}^{2}$ and a slice thickness of 4-5 mm. ${ }^{16}$ An additional diffusion-weighted echo planar imaging sequence was acquired with b0 as the lowest and b1000 s/mm $\mathrm{m}^{2}$ as the highest b-factor, an in plane resolution of 7.8-9.6 $\mathrm{mm}^{2}$ and a slice thickness of 4-5 mm, as described in previous reports from the participating centers. ${ }^{17-19}$

\section{Image Evaluation}

All images were independently analysed by three readers, who were blinded to all clinical information, other imaging results, and histopathology. Reader 1 (RGHB) was a gastrointestinal (GI) radiologist who was highly specialized with 13 years of experience in reading pelvic MRI. Reader 2 (FCHB) was a GI radiologist with 3 years of experience in reading pelvic MRI. Reader 3 (VV) was a GI radiologist with 2 years pelvic MRI expertise and 5 years of experience in reading DWI images in head and neck, abdominal cancer, and lymphoma. The three readers first evaluated the standard postchemoradiation (restaging) MR images and scored the likelihood of a complete response of the primary tumor using a confidence level score $(0=$ definitely residual tumor, $1=$ probably residual tumor, 2 = possibly residual tumor/possibly complete response, 3 = probably complete response, $4=$ definitely complete response). The pre-CRT images were at the readers' disposal to identify the primary tumor, which is just like the evaluation process performed in daily clinical practice. Subsequently, the confidence level-based scoring of the restaging MRI was repeated after addition of the b1000 DWI images.

\section{Imaging Criteria}

On standard MRI, a normalized rectal wall without any detectable wall thickening was considered a definite 
FIG. 1 Standard T2-weighted images of a female patient with a tumor $(T)$ in the mid-rectum, before (a) and after (b) preoperative chemoradiation therapy. After chemoradiation, the tumor has completely disappeared and a normalized rectal wall can be visualized (arrowheads). This feature was considered strongly predictive for a complete tumor response
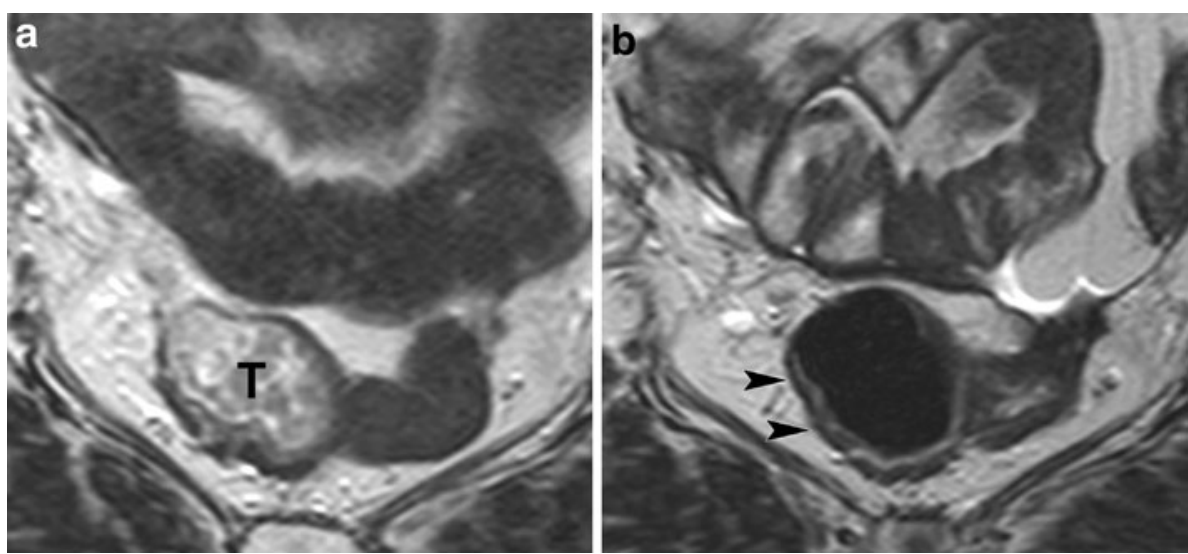

FIG. 2 Standard T2-weighted images of a male patient with a tumor $(T)$ in the rectum, before (a) and after

(b) preoperative chemoradiation therapy. After chemoradiation, a solid residual tumor mass is still visualized (arrow). This feature was considered strongly predictive for the presence of residual tumor
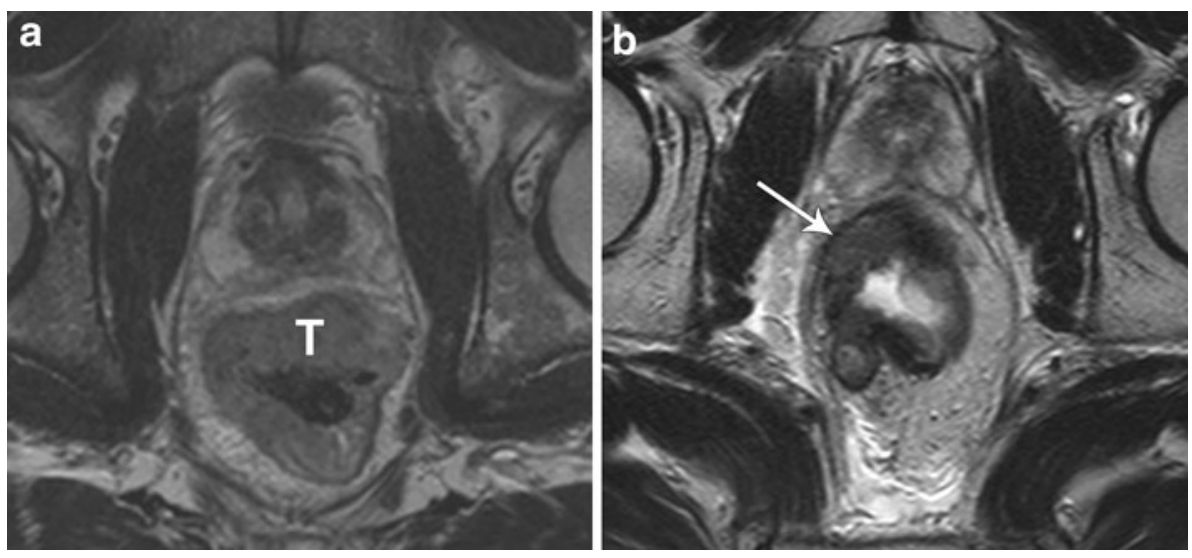

criterion for a complete response (Fig. 1). A solid residual mass with intermediate signal intensity on T2-weighted MRI was considered a definite criterion for residual tumor (Fig. 2). Hypointense signal intensity changes indicated fibrosis, in which case undetermined scores were assigned (Fig. 3). ${ }^{18}$ On the diffusion images, residual high-signal intensity on the location of the primary tumor was considered a criterion for residual tumor, whereas the absence of increased signal on DWI was indicative of a complete response (Fig. 3). The readers assigned a confidence level 2 score (equivocal score) when they were not able to differentiate between a complete response or residual tumor.

\section{Reference Standard}

Histopathologic evaluation of the surgical resection specimen, according to the TNM staging system, served as the reference standard. The tumor regression grade (TRG) was evaluated according to the method of Mandard..$^{20}$ The response of the primary tumor to chemoradiation was graded as follows: "pathologic complete response" (= ypT0/ TRG 1, no residual tumor cells) or "residual tumor" (= ypT1-4 / TRG 2-5, varying from limited tumor cells to a solid residual tumor mass). Eight patients did not undergo surgery, due to strong clinical evidence of a complete response (repeated negative sigmoidoscopy and biopsies after CRT). For these eight patients, a local and distant recurrence-free follow-up period of $>24$ months was considered a surrogate endpoint for a complete response.

\section{Statistical Analysis}

Statistical analyses were performed using the Statistical Package for the Social Sciences (SPSS, version 16.0, Inc., Chicago, IL). Receiver operator characteristics (ROC) curve analyses were performed to evaluate the diagnostic performance of (1) standard MRI only and (2) standard MRI + DWI for identification of a complete response. Corresponding areas under the ROC curve (AUC), sensitivities, specificities, positive predictive values (PPV), and negative predictive values (NPV) with $95 \%$ confidence intervals (CI) were calculated. For these analyses, it had been decided at the start of the study to dichotomize the confidence level scores between 2 (possibly residual tumor/ possibly complete response) and 3 (probably complete response). Differences in diagnostic performance between standard MRI only and the combination of standard MRI + DWI were analyzed by comparing the ROC curves 

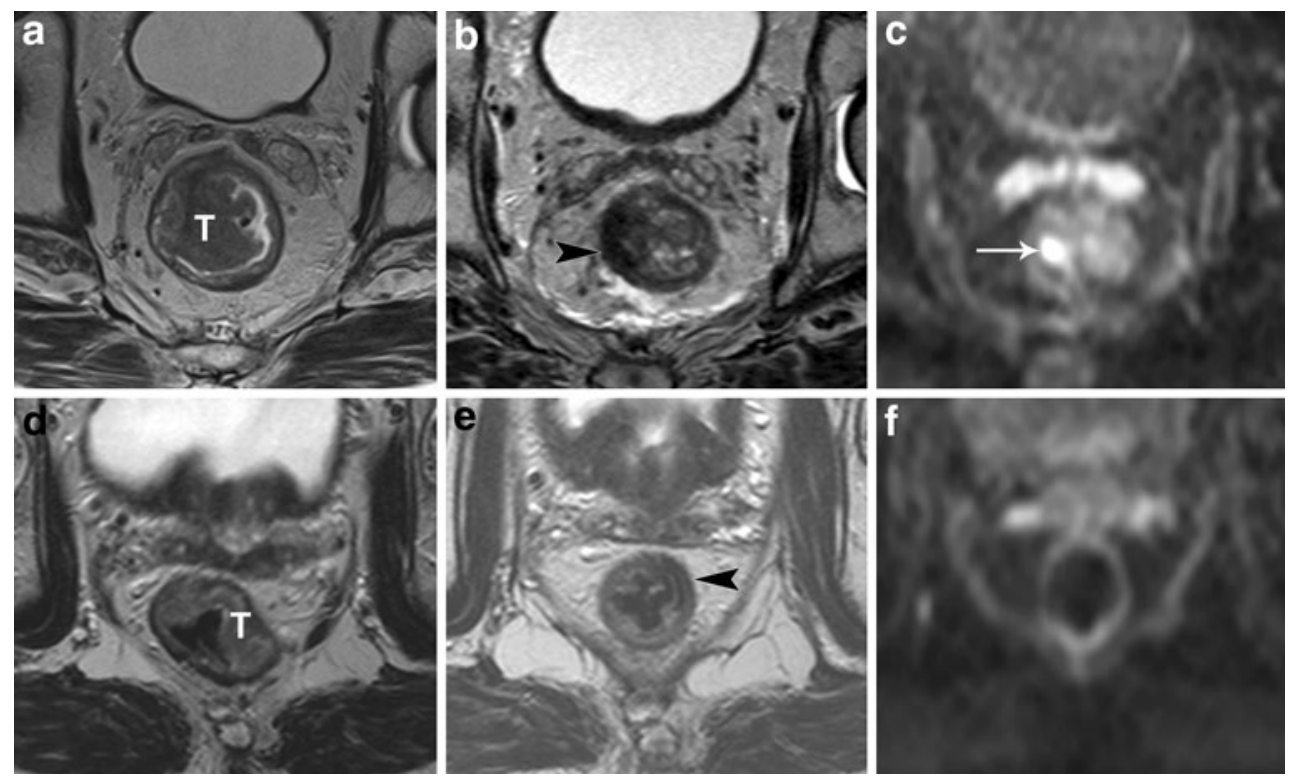

FIG. 3 Standard T2-weighted images of two patients with a tumor $(T)$ in the rectum before $(\mathbf{a}, \mathbf{d})$ and after chemoradiation treatment $(\mathbf{b}$, e). In both cases, the tumor bed has become fibrotic after chemoradiation (arrowheads), which makes it difficult to discriminate between residual tumor and a complete response. In the upper

according to the method described by DeLong et al. ${ }^{21}$ $P$ values $<0.05$ were considered statistically significant. Weighted kappa values with quadratic kappa weighting (0-0.2 poor, 0.21-0.4 fair, 0.41-0.6 moderate, 0.61-0.8 good and $0.81-1$ excellent agreement) were calculated to evaluate interobserver variability. ${ }^{22}$

\section{RESULTS}

\section{Patient and Treatment Characteristics}

A total of 79 patients underwent low anterior resection, 25 had abdominoperineal resection, 4 had more extended surgery, and 4 had local excision (transanal endoscopic microsurgery). At histology, 17 patients had ypT0, 11 had ypT1, 25 had ypT2, 55 had ypT3 and 4 had ypT4 status. Ten patients had mucinous type adenocarcinoma. The median time interval between the restaging MRI and surgery was 15 (range 0-61) days. The eight patients who did not undergo surgery had a median local and distant recurrence-free follow-up of 42.5 (range 26-73) months; these patients were therefore considered complete responders. All together, 25 patients had a complete response and 95 had residual tumor. Of the patients with residual tumor, 63 were $\mathrm{yN} 0,22$ were $\mathrm{yN} 1$, and 10 were yN2 status. Of the patients with a complete tumor response, 23 were $\mathrm{yN} 0,1$ was $\mathrm{yN} 1$, and 1 was yN2 status. There were no significant differences in patient characteristics, gender, or age distribution between the separate centers. patient, there is still a clear high signal intensity area on DWI (arrow in c), which was confirmed to be a ypT2 residual tumor at histology. In the lower patient, no high signal is shown on DWI (f) and a complete tumor response (ypT0) was confirmed at histology

\section{Diagnostic Performance for Selection of Complete Responders}

ROC curves for the selection of complete responders are displayed in Fig. 4. Corresponding accuracy figures and AUCs with $95 \%$ confidence intervals are provided in Table 1 . For the highly expert reader 1 , AUC improved from 0.76 for standard MRI to 0.8 for standard MRI + DWI $(P=0.39)$. For the less experienced reader 2, AUC improved from 0.68 on standard MRI to 0.8 after addition of DWI $(P=0.02)$. For reader 3, AUC improved from 0.58 on standard MRI to 0.78 after addition of DWI $(P=0.002)$.

\section{Number of Equivocal (Confidence Level 2) Scores}

When using only standard MRI without DWI, readers 1, 2 , and 3 assigned a confidence level score of 2 (possibly residual tumor/possibly complete response) to 31 , 7, and 41 patients, respectively. After addition of DWI, the number of equivocal scores decreased to 2,4 , and 2 for the three readers, respectively. This resulted in a reduced number of false negatives for prediction of a complete tumor response, ranging from 9-12 for the three readers on standard MRI + DWI compared with $15-25$ on standard MRI only. The number of false positives remained unchanged and ranged from 2-8 on standard MRI and from 3-10 after addition of DWI. 

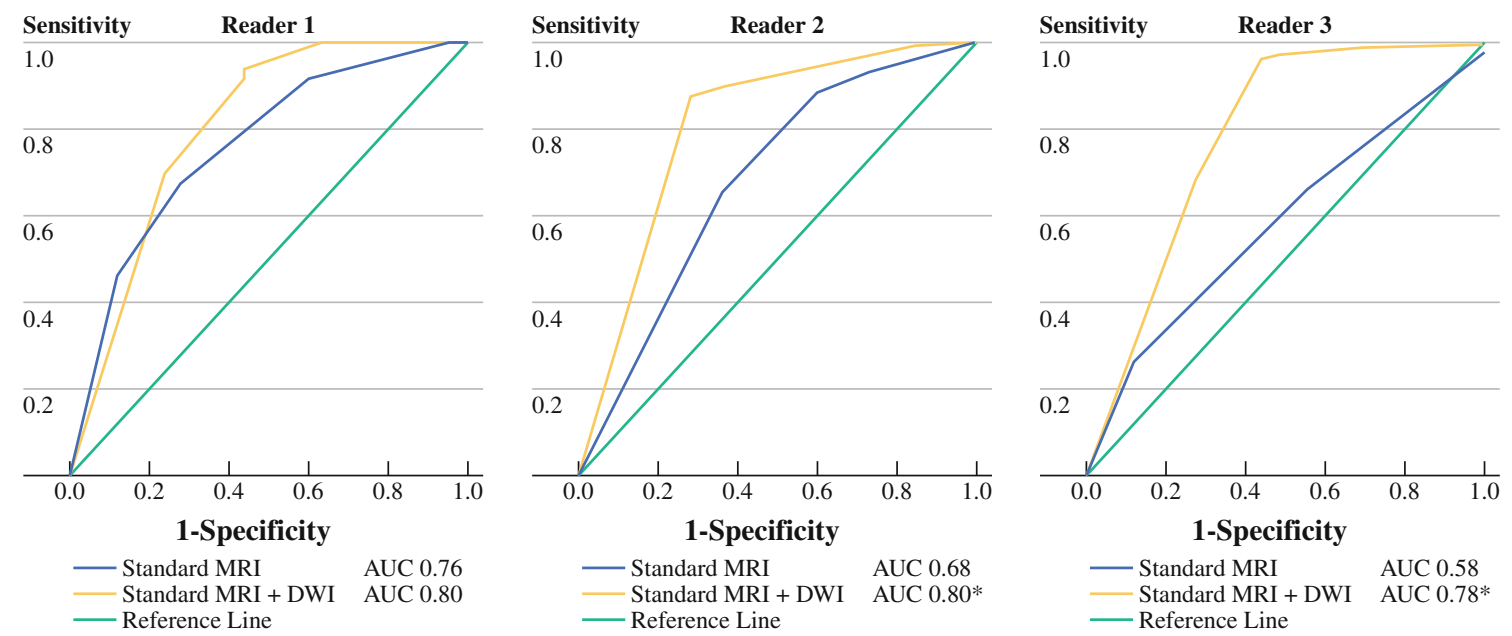

FIG. 4 Receiver operator characteristics curves and areas under the curve $(A U C)$ of the three readers for identification of a complete tumor response after CRT using only standard MRI and standard
MRI + DWI, respectively. Diagnostic performance improved significantly $(*)$ for reader $2(P=0.02)$ and reader $3(P=0.002)$. For reader 1 , there was no significant improvement $(P=0.39)$

TABLE 1 Diagnostic performance for the prediction of a complete response (ypT0)

\begin{tabular}{|c|c|c|c|c|c|c|}
\hline & \multicolumn{3}{|c|}{ Standard MRI only } & \multicolumn{3}{|c|}{ Standard MRI + DWI } \\
\hline & $\mathrm{R} 1$ & $\mathrm{R} 2$ & R3 & $\mathrm{R} 1$ & $\mathrm{R} 2$ & R3 \\
\hline Sensitivity & $40(10 / 25)$ & $28(7 / 25)$ & $0(0 / 25)$ & $56(14 / 25)$ & $64(16 / 25)$ & $52(13 / 25)$ \\
\hline $95 \% \mathrm{CI}$ & $26-53$ & $16-40$ & $0-0$ & $41-67$ & $48-77$ & $39-60$ \\
\hline Specificity & $92(87 / 95)$ & $93(88 / 95)$ & $98(93 / 95)$ & $94(89 / 95)$ & $89(85 / 95)$ & $97(92 / 95)$ \\
\hline $95 \% \mathrm{CI}$ & $88-95$ & $89-96$ & $98-99$ & $90-97$ & $85-93$ & $93-99$ \\
\hline PPV & $56(10 / 18)$ & $50(7 / 14)$ & $0(0 / 2)$ & $70(14 / 20)$ & $62(16 / 26)$ & $81(13 / 16)$ \\
\hline $95 \% \mathrm{CI}$ & $36-73$ & $28-71$ & $0-0$ & $52-84$ & $46-74$ & $60-93$ \\
\hline NPV & $85(87 / 102)$ & $83(88 / 106)$ & $79(93 / 118)$ & $89(89 / 100)$ & $90(85 / 94)$ & $88(92 / 104)$ \\
\hline $95 \% \mathrm{CI}$ & $82-88$ & $80-86$ & $79-80$ & $85-92$ & $86-94$ & $85-90$ \\
\hline AUC & 0.76 & 0.68 & 0.58 & 0.80 & 0.80 & 0.78 \\
\hline $95 \% \mathrm{CI}$ & $0.65-0.86$ & $0.56-0.8$ & $0.47-0.69$ & $0.69-0.91$ & $0.7-0.91$ & $0.67-0.9$ \\
\hline
\end{tabular}

$R 1$ reader 1, GI radiologist with 13 years experience in pelvic MRI; $R 2$ reader 2, GI radiologist with 3 years experience in pelvic MRI; $R 3$ reader 3 , GI radiologist with 2 years experience in pelvic MRI and 5 years experience in reading DWI; $P P V$ positive predictive value; $N P V$ negative predictive value; $A U C$ area under the ROC curve; $C I$ confidence interval

Numbers are percentages; absolute numbers are given in parentheses

\section{Interobserver Agreement}

Kappa values for the interobserver agreement between the three readers are displayed in Table 2. Interobserver agreement improved from fair agreement $(\kappa 0.2-0.32)$ on standard MRI to moderate agreement $(\kappa 0.51-0.55)$ after addition of DWI.

\section{DISCUSSION}

The findings of this study indicate that the diagnostic performance for predicting a pathologic complete tumor response after chemoradiation improved for the combination of standard MRI + DWI (AUC 0.78-0.8) compared
TABLE 2 Interobserver agreement between the three readers

\begin{tabular}{lll}
\hline Observers & Standard MRI only $(\kappa)$ & Standard MRI + DWI $(\kappa)$ \\
\hline R1 and R2 & 0.32 & 0.55 \\
R1 and R3 & 0.31 & 0.52 \\
R2 and R3 & 0.2 & 0.51
\end{tabular}

$R 1$ reader 1 , GI radiologist with 13 years experience in pelvic MRI; $R 2$ reader 2, GI radiologist with 3 years experience in pelvic MRI; $R 3$ reader 3, GI radiologist with 2 years experience in pelvic MRI and 5 years experience in reading DWI

Kappa values are weighted kappa's with quadratic kappa weighting

with standard MRI only (AUC $0.58-0.76$ ). With the addition of DWI, sensitivity for identification of a complete response improved by $16-52 \%$ for the three readers. 
Moreover, it resulted in a substantial reduction in the number of equivocal scores and an improved interobserver agreement.

Of interest is the improved sensitivity for the combination of MRI + DWI; i.e., it resulted in less overestimation of tumor in patients with a complete tumor response. This is mainly because on the restaging MRI without DWI many interpretation difficulties were observed when the primary tumor bed had become fibrotic as a result of the radiation treatment. In these cases, it is difficult to differentiate small areas of residual tumor from mere fibrosis and readers tend to overestimate the presence of tumor (Fig. 3). ${ }^{23-26}$ Apparently, this is where the functional information from DWI proves beneficial. Areas of fibrosis typically have a low cellular density, which results in low signal intensity on high b-value (b1000) diffusion images. ${ }^{27}$ Conversely, residual tumor areas have a relatively high cellular density and show high signal on DWI, which stands out within the low signal of the surrounding tissue/fibrosis. This is the reason why small areas of residual tumor are better depicted on DWI. ${ }^{8,27}$ Nevertheless, interpretation errors were still observed with DWI resulting in a suboptimal sensitivity of $52-64 \%$. When the signal of the normal rectal wall is not fully suppressed on DWI, which often occurs when the rectal wall is collapsed, high signal at the location of the initial tumor area may erroneously be interpreted as residual tumor, resulting in overstaging errors. In addition, some imaging artifacts may occur on DWI, particularly around air-tissue interfaces. It is relevant to recognize these shortcomings of DWI and initiate teaching courses in which radiologists will be trained in the interpretation of DWI and will become familiar with its pitfalls. Specificity for MRI and DWI is $>90 \%$, indicating that the residual tumors are accurately detected and the risk for undertreatment will be $<10 \%$. Although DWI allows detection of even small (2-5 $\mathrm{mm}$ ) tumor volumes, the challenge will remain the detection of microscopically small clusters of residual tumor cells, which are difficult to detect-even at histology-and are currently beyond the detection level of any available imaging modality, including DWI.

The addition of DWI improved the performance of all readers, albeit that this benefit was not significant for reader 1 . His extensive experience of 13 years in interpreting rectal cancer MRI may explain why reader 1 was already more accurate with the use of only standard MRI (AUC 0.76). This exceptionally high level of expertise does not reflect common daily practice. Our study, however, clearly shows that for radiologists in general centers with expertise levels like the other two readers, DWI can really be of value. Furthermore, all readers showed a significant reduction in equivocal (confidence level 2) scores after addition of DWI, indicating that it raised their confidence in the discrimination between complete responders and residual tumor. This also explains the better interobserver agreement between the readers after addition of DWI.

So far, the largest body of evidence for response evaluation exists for ${ }^{18}$ FDG-PET. Changes in FDG uptake, in particular early $( \pm 2$ weeks $)$ after onset of treatment, have proven useful for prediction of response. ${ }^{4,28,29}$ PET is, however, less reliable in identifying the complete tumor responders after completion of chemoradiation: up to $55 \%$ of the residual tumors are overlooked and patients are erroneously interpreted as complete responders. ${ }^{5,6,28}$ In a recent study by Janssen et al., only one of six complete responders as identified on FDG-PET corresponded with a true complete response at histology. ${ }^{4}$ When using FDGPET for treatment planning, the main risk would be an undertreatment of these patients. In our DWI-MRI study, the presence of residual tumor was underestimated in only $<10 \%$ of the cases, indicating that-compared to PETthere is a considerably smaller risk for undertreatment.

To the best of our knowledge, this is the largest and only multicenter study to investigate the value of DWI for identifying complete tumor responders after CRT for rectal cancer. It confirms previous findings of a smaller, singlecenter study by Kim et al. ${ }^{8}$ Previous studies also have shown promise for quantitative DWI measurements of the "apparent diffusion coefficient" (ADC) (performed before, during, and/or after chemoradiation treatment) to predict the degree of response to therapy.,10-15,17,30,31 In our study, we only focussed on qualitative, visual evaluation of DWI and did not quantitatively measure ADC. This is a more convenient approach, because a visual analysis is more practical and less time-consuming for a busy radiology practice. Furthermore, ADC values are dependent on technical variations among DWI sequences generated by different MR equipment. ADC data from multiple centers may be less suitable for pooled analysis. Visual evaluation of DWI images is less subjected to technical variations, and pooling of these data was feasible because all three participating centers acquired a DWI sequence with equal (b1000) diffusion weighting. Nevertheless, we acknowledge that small variations between the participating centers may have introduced some bias.

In the current study, we only focused on response assessment of the primary tumor and not the lymph nodes. The prevalence of a positive lymph node status in case of a complete response of the primary tumor after CRT is very low and was only 2 of $25(8 \%)$ in the present study. Nevertheless, to safely offer patients a wait-and-see policy after CRT, we have to ensure that both the primary tumor and all metastatic nodes have undergone a complete regression (ypTON0). Although standard MRI is known to be inaccurate for the primary staging of rectal cancer nodes, ${ }^{32,33}$ there is evidence that after chemoradiation, 
MRI performs considerably better. High NPVs ranging from $81-100 \%$ have been reported, suggesting that the ypNO patients can already be accurately selected and the addition of functional techniques, such as DWI, may not even be necessary. ${ }^{7,19,23,34-36}$ Furthermore, the only study to focus specifically on DWI for staging of rectal cancer nodes after CRT already showed good results for standard MRI only (NPV 94-95\%) and reported no clear benefit after addition of DWI (NPV 92-93\%). The main role of DWI for lymph node evaluation was that it improved the number of detected nodes (both benign and malignant), because nodes were more easily detected on DWI due to their high signal intensity compared with the suppressed background signal of surrounding tissues. ${ }^{19}$

\section{Clinical Impact}

A wait-and-see approach ${ }^{3}$ or local excision ${ }^{37}$ for patients with a good response after chemoradiation is at present still debatable. Initiating and performing large patient studies to prove their efficiency is difficult, partly because clinicians are not convinced that safe selection of the right patients can be done. Therefore, one of the most important cornerstones to make implementation of such minimally invasive treatments possible is a precise selection of the eligible patients. Our goal was to assess whether MR imaging can be beneficial in this regard. Because of its reported promise in cancer imaging, we particularly looked at the potential of diffusion-weighted MRI. Moreover, DWI is a noninvasive technique that does not require the use of ionizing radiation or contrast agents and can easily be added to any standard MRI protocol. Our results suggest that, by combining morphological with functional imaging information, MRI + DWI can significantly improve sensitivity for selection of complete responders. Furthermore, specificity is $>90 \%$, which indicates that the risk for underestimation of residual tumor can be brought to $<10 \%$. As an adjunct to clinical tools (digital examination, endoscopy, and biopsy), the combined use of MRI + DWI seems promising to enable a more precise selection of patients eligible to undergo minimally invasive treatments. The current results are obviously still premature for clinical decision-making, but its promise warrants further large and prospective patient studies.

In conclusion, this study shows that the addition of diffusion-weighted imaging to a standard, restaging MRI improves the performance and confidence of radiologists in selecting the patients with a pathological complete tumor response after chemoradiation for locally advanced rectal cancer. The combination of MRI + DWI could be of additional value for the clinical assessment of these patients.
ACKNOWLEDGMENT The authors thank Frederik de Keyzer for his assistance with the statistical data analyses.

DISCLOSURE None of the authors have any commercial interest to disclose.

OPEN ACCESS This article is distributed under the terms of the Creative Commons Attribution Noncommercial License which permits any noncommercial use, distribution, and reproduction in any medium, provided the original author(s) and source are credited.

\section{REFERENCES}

1. Sauer R, Becker H, Hohenberger W, et al. German Rectal Cancer Study Group. Preoperative versus postoperative chemoradiotherapy for rectal cancer. $N$ Engl J Med. 2004;351:1731-40.

2. Maas M, Nelemans $P$, Valentini V, et al. Long-term outcome in patients with a pathological complete response after chemoradiation for rectal cancer: a pooled analysis of individual patient data. Lancet Oncol. 2010;11:835-44.

3. Habr-Gama A, Perez RO, Proscurshim I, Campos FG, Nadalin W, Kiss D, Gama-Rodrigues J. Patterns of failure and survival for nonoperative treatment of stage $\mathrm{c} 0$ distal rectal cancer following neoadjuvant chemoradiation therapy. J Gastrointest Surg. 2006; 10:1319-28; discussion 28-9.

4. Janssen MH, Ollers MC, Riedl RG, et al. Accurate prediction of pathological rectal tumor response after two weeks of preoperative radiochemotherapy using (18)F-fluorodeoxyglucose-positron emission tomography-computed tomography imaging. Int $J$ Radiat Oncol Biol Phys. 2010;77:392-9.

5. Capirci C, Rubello D, Chierichetti F, et al. Restaging after neoadjuvant chemoradiotherapy for rectal adenocarcinoma: role of F18-FDG PET. Biomed Pharmacother. 2004;58:451-7.

6. Kristiansen C, Loft A, Berthelsen AK, Graff J, Lindebjerg J, Bisgaard C, Jakobsen A. PET/CT and histopathologic response to preoperative chemoradiation therapy in locally advanced rectal cancer. Dis Colon Rectum. 2008;51:21-5.

7. Suppiah A, Hunter IA, Cowley J, Garimella V, Cast J, Hartley JE, Monson JR. Magnetic resonance imaging accuracy in assessing tumour down-staging following chemoradiation in rectal cancer. Colorectal Dis. 2009;11:249-53.

8. Kim SH, Lee JM, Hong SH, Kim GH, Lee JY, Han JK, Choi BI. Locally advanced rectal cancer: added value of diffusionweighted MR imaging in the evaluation of tumor response to neoadjuvant chemo- and radiation therapy. Radiology. 2009; 253:116-25.

9. Vanagunas A, Lin DE, Stryker SJ. Accuracy of endoscopic ultrasound for restaging rectal cancer following neoadjuvant chemoradiation therapy. Am J Gastroenterol. 2004;99:109-12.

10. Sun YS, Zhang XP, Tang L, Ji JF, Gu J, Cai Y, Zhang XY. Locally advanced rectal carcinoma treated with preoperative chemotherapy and radiation therapy: preliminary analysis of diffusion-weighted MR imaging for early detection of tumor histopathologic downstaging. Radiology. 2010;254:170-8.

11. DeVries AF, Kremser C, Hein PA, Griebel, et al. Tumor microcirculation and diffusion predict therapy outcome for primary rectal carcinoma. Int J Radiat Oncol Biol Phys. 2003; 56:958-65.

12. Hein PA, Kremser C, Judmaier W, et al. Diffusion-weighted magnetic resonance imaging for monitoring diffusion changes in rectal carcinoma during combined, preoperative chemoradiation: preliminary results of a prospective study. Eur J Radiol. 2003; $45: 214-22$. 
13. Dzik-Jurasz A, Domenig C, George M, Wolber J, Padhani A, Brown G, Doran S . Diffusion MRI for prediction of response of rectal cancer to chemoradiation. Lancet. 2002;360:307-8.

14. Patterson DM, Padhani AR, Collins DJ. Technology insight: water diffusion MRI-a potential new biomarker of response to cancer therapy. Nat Clin Pract Oncol. 2008;5:220-33.

15. Kremser C, Judmaier W, Hein P, Griebel J, Lukas P, de Vries A. Preliminary results on the influence of chemoradiation on apparent diffusion coefficients of primary rectal carcinoma measured by magnetic resonance imaging. Strahlenther Onkol. 2003;179:641-9.

16. Brown G, Kirkham A, Williams GT, et al. High-resolution MRI of the anatomy important in total mesorectal excision of the rectum. AJR Am J Roentgenol. 2004;182:431-9.

17. Lambrecht M, Deroose C, Roels S, et al. The use of FDG-PET/ $\mathrm{CT}$ and diffusion-weighted magnetic resonance imaging for response prediction before, during and after preoperative chemoradiotherapy for rectal cancer. Acta Oncol. 2010;49:956-63.

18. Barbaro B, Vitale R, Leccisotti L, et al. Restaging locally advanced rectal cancer with MR imaging after chemoradiation therapy. Radiographics. 2010;30:699-716.

19. Lambregts DMJ, Maas M, Riedl RG, et al. Value of ADC measurements for nodal staging after chemoradiation in locally advanced rectal cancer-a per lesion validation study. Eur Radiol. 2010;21:265-73.

20. Mandard AM, Dalibard F, Mandard JC, et al. Pathologic assessment of tumor regression after preoperative chemoradiotherapy of esophageal carcinoma. Clinicopathologic correlations. Cancer. 1994;73:2680-6.

21. DeLong ER, DeLong DM, Clarke-Pearson DL. Comparing the areas under two or more correlated receiver operating characteristic curves: a nonparametric approach. Biometrics. 1988;44: 837-45.

22. Cohen J. Weighted kappa: nominal scale agreement with provision for scaled disagreement or partial credit. Psychol Bull. 1968;70:213-20.

23. Barbaro B, Fiorucci C, Tebala C, et al. Locally advanced rectal cancer: MR imaging in prediction of response after preoperative chemotherapy and radiation therapy. Radiology. 2009;250:730-9.

24. Kuo LJ, Chern MC, Tsou MH, et al. Interpretation of magnetic resonance imaging for locally advanced rectal carcinoma after preoperative chemoradiation therapy. Dis Colon Rectum. 2005;48:23-8.

25. Dresen RC, Beets GL, Rutten HJ, et al. Locally advanced rectal cancer: MR imaging for restaging after neoadjuvant radiation therapy with concomitant chemotherapy. Part I. Are we able to predict tumor confined to the rectal wall? Radiology. 2009;252:71-80.

26. Jonas J, Bahr R. Neoadjuvant chemoradiation treatment impairs accuracy of MRI staging in rectal carcinoma. Gut. 2006;55:1214-5.
27. Vandecaveye V, De Keyzer F, Nuyts S, et al. Detection of head and neck squamous cell carcinoma with diffusion weighted MRI after (chemo)radiotherapy: correlation between radiologic and histopathologic findings. Int J Radiat Oncol Biol Phys. 2007;67: 960-71.

28. de Geus-Oei LF, Vriens D, van Laarhoven HW, van der Graaf WT, Oyen WJ. Monitoring and predicting response to therapy with 18F-FDG PET in colorectal cancer: a systematic review. J Nucl Med. 2009;50(Suppl 1):43S-54S.

29. Cascini GL, Avallone A, Delrio P, et al. 18F-FDG PET is an early predictor of pathologic tumor response to preoperative radiochemotherapy in locally advanced rectal cancer. $\mathrm{J} \mathrm{Nucl}$ Med. 2006;47:1241-8.

30. Roth Y, Tichler T, Kostenich G, et al. High-b-value diffusionweighted MR imaging for pretreatment prediction and early monitoring of tumor response to therapy in mice. Radiology. 2004;232:685-92.

31. Kim SH, Lee JY, Lee JM, Han JK, Choi BI. Apparent diffusion coefficient for evaluating tumour response to neoadjuvant chemoradiation therapy for locally advanced rectal cancer. Eur Radiol. 2010;Oct 27 [Epub ahead of print].

32. Bipat S, Glas AS, Slors FJ, Zwinderman AH, Bossuyt PM, Stoker J. Rectal cancer: local staging and assessment of lymph node involvement with endoluminal US, CT, and MR imaging: a metaanalysis. Radiology. 2004;232:773-83.

33. Lahaye MJ, Engelen SM, Nelemans PJ, Beets GL, van de Velde CJ, van Engelshoven JM, Beets-Tan RG. Imaging for predicting the risk factors-the circumferential resection margin and nodal disease-of local recurrence in rectal cancer: a meta-analysis. Semin Ultrasound CT MR. 2005;26:259-68.

34. Lahaye MJ, Beets GL, Engelen SM, et al. Locally advanced rectal cancer: MR imaging for restaging after neoadjuvant radiation therapy with concomitant chemotherapy. Part II. What are the criteria to predict involved lymph nodes? Radiology. 2009; 252:81-91.

35. Maretto I, Pomerri F, Pucciarelli S, et al. The potential of restaging in the prediction of pathologic response after preoperative chemoradiotherapy for rectal cancer. Ann Surg Oncol. 2007; 14:455-61.

36. Denecke T, Rau B, Hoffmann KT, et al. Comparison of CT, MRI and FDG-PET in response prediction of patients with locally advanced rectal cancer after multimodal preoperative therapy: is there a benefit in using functional imaging? Eur Radiol. 2005;15:1658-66.

37. Lezoche G, Baldarelli M, Guerrieri M, Paganini AM, De Sanctis A, Bartolacci S, Lezoche E. A prospective randomized study with a 5-year minimum follow-up evaluation of transanal endoscopic microsurgery versus laparoscopic total mesorectal excision after neoadjuvant therapy. Surg Endosc. 2008;22:352-8. 